\title{
Category of Submodules of a Uniserial Module
}

\author{
Fitriani $^{1, *}$, Indah Emilia Wijayanti ${ }^{2}$, Budi Surodjo $^{2}$, Sri Wahyuni $^{2}$, Ahmad Faisol $^{1}$ \\ ${ }^{1}$ Department of Mathematics, Universitas Lampung, Lampung, Indonesia \\ ${ }^{2}$ Department of Mathematics, Universitas Gadjah Mada, Yogyakarta, Indonesia
}

Received June 29, 2021; Revised August 24, 2021; Accepted August 30, 2021

Cite This Paper in the following Citation Styles

(a): [1] Fitriani, Indah Emilia Wijayanti, Budi Surodjo, Sri Wahyuni, Ahmad Faisol, "Category of Submodules of a Uniserial Module," Mathematics and Statistics, Vol.9, No.5, pp. 744-748, 2021. DOI: 10.13189/ms.2021.090514

(b): Fitriani, Indah Emilia Wijayanti, Budi Surodjo, Sri Wahyuni, Ahmad Faisol, (2021). Category of Submodules of a Uniserial Module. Mathematics and Statistics, 9(5), 744-748. DOI: 10.13189/ms.2021.090514

Copyright $@ 2021$ by authors, all rights reserved. Authors agree that this article remains permanently open access under the terms of the Creative Commons Attribution License 4.0 International License

\begin{abstract}
Let $R$ be a ring, $K, M$ be $R$-modules, $L$ a uniserial $R$-module, and $X$ a submodule of $L$. The triple $(K, L, M)$ is said to be $X$-sub-exact at $L$ if the sequence $K \rightarrow X \rightarrow M$ is exact. Let $\sigma(K, L, M)$ is a set of all submodules $Y$ of $L$ such that $(K, L, M)$ is $Y$-sub-exact. The sub-exact sequence is a generalization of an exact sequence. We collect all triple $(K, L, M)$ such that $(K, L, M)$ is an $X$-sub exact sequence, where $X$ is a maximal element of $\sigma(K, L, M)$. In a uniserial module, all submodules can be compared under inclusion. So, we can find the maximal element of $\sigma(K, L, M)$. In this paper, we prove that the set $\sigma(K, L, M)$ form a category, and we denoted it by $\mathcal{C}_{L}$. Furthermore, we prove that $\mathcal{C}_{Y}$ is a full subcategory of $\mathcal{C}_{L}$, for every submodule $Y$ of $L$. Next, we show that if $L$ is a uniserial module, then $\mathcal{C}_{L}$ is a pre-additive category. Every morphism in $\mathcal{C}_{L}$ has kernel under some conditions. Since a module factor of $L$ is not a submodule of $L$, every morphism in a category $\mathcal{C}_{L}$ does not have a cokernel. So, $\mathcal{C}_{L}$ is not an abelian category. Moreover, we investigate a monic $X$-sub-exact and an epic $X$-sub-exact sequence. We prove that the triple $(K, L, M)$ is a monic $X$-sub-exact if and only if the triple $\mathbb{Z}$-modules $\quad\left(\operatorname{Hom}_{R}(N, K), \operatorname{Hom}_{R}(N, L), H_{R}(N, M)\right)$ is a monic $\operatorname{Hom}_{R}(N, X)$-sub-exact sequence, for all $R$-modules $N$. Furthermore, the triple $(K, L, M)$ is an epic $X$-sub-exact if and only if the triple $\mathbb{Z}$-modules $\left(\operatorname{Hom}_{R}(M, N), \operatorname{Hom}_{R}(L, N), \operatorname{Hom}_{R}(K, N)\right)$ is a monic $H_{R}(X, N)$-sub-exact, for all $R$-module $N$.
\end{abstract}

Keywords Sub-exact Sequences, Pre-additive Category, Uniserial Module

\section{Introduction}

Let $R$ be a ring and let $A \stackrel{f}{\rightarrow} B \stackrel{g}{\rightarrow} C$ be an exact sequence of $R$-modules, i.e. $\operatorname{Im} f=\operatorname{Ker} g\left(=g^{-1}(0)\right)$. Davvaz and Parnian-Garamaleky [5] generalize this concept to be a quasiexact sequence. A sequence of $R$-modules $A \stackrel{f}{\rightarrow} B \stackrel{g}{\rightarrow} C$ is quasi-exact in $B$ or $U$-exact in $B$ if there exists a submodule $U$ in $C$ such that $\operatorname{Im} f=g^{-1}(U)$.

Davvaz and ShabaniSolt give new basic properties of the $U$-homological algebra [4]. In 2002, Anvariyeh and Davvaz introduced $U$-split sequences and provided several connections between $U$-split sequences, and projective modules [3]. Then, Anvariyeh dan Davvaz give a generalization of Schanuel Lemma and proved further results about quasi-exact sequences [2].

In 2016, Fitriani et al. [7] introduce an $X$-sub-exact sequence as a generalization of an exact sequence. Let $K, L, M$ be $R$-modules and $X$ a submodule of $L$. The triple $(K, L, M)$ is said to be $X$-sub-exact at $L$ if $K \rightarrow X \rightarrow M$ is exact. The exact sequence is a special case of $X$-sub-exact sequence [7]. As an application of a sub-exact sequence, Fitriani et al. introduce an X-sub-linearly independent module [8]. Then, by using the concept of coexact sequence, Fitriani et al. establish a $\mathcal{U}_{V}$-generated module [10]. This concept is a generalization of the $\mathcal{U}$-generated module [13]. Furthermore, they introduce $\mathcal{U}$-basis and $\mathcal{U}$-free modules [9]. Besides that, the sub-exact sequences can be applied in determining the Noetherian property of the submodule of the generalized power series module [6].

Let $\sigma(K, L, M)$ is a set of all submodules $Y$ of $L$ such that $(K, L, M)$ is $Y$-sub-exact. In general, if $Y_{1}$ and $Y_{2}$ are in $\sigma(K, L, M)$, we can not compare $Y_{1}$ and $Y_{2}$ by inclusion. However, if $\mathrm{L}$ is a uniserial module, then any two submodules are comparable concerning inclusion. So, we can find a maximal element of $\sigma(K, L, M)$. 
Let $L$ be a uniserial module. The collection of all triple $(K, L, M)$ such that $(K, L, M)$ is an $X$-sub exact sequence, where $X$ is a maximal element of $\sigma(K, L, M)$ form a category, and we denoted it by $\mathcal{C}_{L}$. In this paper, we will prove that $\mathcal{C}_{Y}$ is a full subcategory of $\mathcal{C}_{L}$, for every submodule $Y$ of $L$. Furthermore, we will show that $\mathcal{C}_{L}$ is a pre-additive category, and every morphism in $\mathcal{C}_{L}$ has kernel under some conditions. We investigate about a monic $X$-sub-exact and an epic $X$-subexact sequences.

Let $K, L, M$ be $R$-modules and $\sigma(K, L, M)=\{X \leq$ $L \mid(K, L, M) X$-sub-exact at $L\}$. Since $0 \in \sigma(K, L, M)$, $\sigma(K, L, M) \neq \emptyset$. The set $\sigma(K, L, M)$ is not closed under submodules. If a submodule $N$ of $L$ is a direct summand of any element of $\sigma(K, L, M), N$ is contained in $\sigma(K, L, M)$.

Let $K, L, M$ be $R$-modules and $X_{1}, X_{2}$ submodules of $L$, where $X_{2} \subset X_{1}$. If $X_{1} \in \sigma(K, L, M)$ and $X_{2}$ is a direct summand of $X_{1}$, then $X_{2} \in \sigma(K, L, M)$ [7]. Therefore, if $L$ is semisimple and $L \in \sigma(K, L, M)$, then any submodule of $L$ is contained in $\sigma(K, L, M)$. Moreover, $\sigma(K, L, M)$ is not closed under extensions.

If there are $R$-module homomorphisms $f$ and $g$ such that the sequence

$$
K \stackrel{f}{\rightarrow} L \stackrel{g}{\rightarrow} M
$$

is exact, then $\sigma(K, L, M)$ has a maximal element. If not, the set $\sigma(K, L, M)$ has a maximal element if $L$ is Noetherian. Furthermore, $\sigma(K, L, M)$ may has more than one maximal element. But, any two elements of $\sigma(K, L, M)$ are not necesarrily unique up to isomorphism [7].

We recall definition of an additive category and an uniserial module as follow: A category $\mathcal{A}$ is called an additive category if the following conditions hold:

(A1) For every pair of objects $X, Y$ the set of morphisms $\operatorname{Hom}_{\mathcal{A}}(X, Y)$ is an abelian group and the composition of morphisms

$$
\operatorname{Hom}_{\mathcal{A}}(Y, Z) \times \operatorname{Hom}_{\mathcal{A}}(X, Y) \rightarrow \operatorname{Hom}_{\mathcal{A}}(X, Z)
$$

is bilinear over the integers.

(A2) $\mathcal{A}$ contains a zero object 0 (i.e. for every object $X \in \mathcal{A}$ each morphism set $\operatorname{Hom}_{\mathcal{A}}(X, 0)$ and $\operatorname{Hom}_{\mathcal{A}}(0, X)$ has precisely one element).

(A3) For every pair of objects $X, Y$ in $\mathcal{A}$ there exists a coproduct $X \oplus Y$ in $\mathcal{A}$.

A category satisfying $(A 1)$ and $(A 2)$ is called a preadditive category [11].

A module $\mathrm{M}$ over any $\operatorname{ring} R$ is uniserial if $M \neq 0$ and the submodules of $M$ form a chain (that is, any two of them are comparable under inclusion) [12].

\section{Main Result}

Let $K, L, M$ be $R$-modules, where $L$ be a uniserial module. We collect all triples $(K, L, M)$ such that $(K, L, M)$ is $X$-subexact, for some submodule $X$ of $L$. We define:

$$
\sigma(K, L, M)=\{X \leq L \mid K \rightarrow X \rightarrow M \text { exact }\}
$$

Let $X_{1}, X_{2} \in \sigma(K, L, M)$. Since $L$ is a uniserial module, we have $X_{1} \subseteq X_{2}$ or $X_{2} \subseteq X_{1}$. So, we have a maximal element in $\sigma(K, L, M)$.

We will show that all triples $(K, L, M)$ such that $(K, L, M)$ is $X$-sub-exact at $L$, where $X$ is a maximal element of $\sigma(K, L, M)$, form a category, we denote it by $\mathcal{C}_{L}$. A maximal element of $\sigma(K, L, M)$ will represent $(K, L, M)$ to be an object in category $\mathcal{C}_{L}$.

Category of $\mathcal{C}_{L}$ is given by:

1. Objects: Class of all triples $(K, L, M)$ such that $(K, L, M)$ is $X$-sub-exact, where $X$ is a maximal element of $\sigma(K, L, M)$.

\section{Morphisms:}

Let $\left(K_{1}, L, M_{1}\right),\left(K_{2}, L, M_{2}\right) \in \operatorname{Obj}\left(\mathcal{C}_{L}\right)$. Then, there exist submodules $X_{1}, X_{2}$ of $L$ and $R$-homomorphisms $f_{1}, g_{1}, f_{2}, g_{2}$ such that the sequences:

$$
K_{1} \stackrel{f_{1}}{\longrightarrow} X_{1} \stackrel{g_{1}}{\longrightarrow} M_{1}
$$

and

$$
K_{2} \stackrel{f_{2}}{\longrightarrow} X_{2} \stackrel{g_{2}}{\longrightarrow} M_{2}
$$

are exact, where $X_{1}$ and $X_{2}$ are maximal element of $\sigma\left(K_{1}, L, M_{1}\right)$ and $\sigma\left(K_{2}, L, M_{2}\right)$, respectively.

A morphism $\theta=(\alpha, \beta, \gamma)$ from $\left(K_{1}, L, M_{1}\right)$ to $\left(K_{2}, L, M_{2}\right)$, where $\alpha: K_{1} \rightarrow K_{2}, \beta: X_{1} \rightarrow X_{2}$ and $\gamma: M_{1} \rightarrow M_{2}$ are $R$-module homomorphisms such that the following diagram with exact rows:

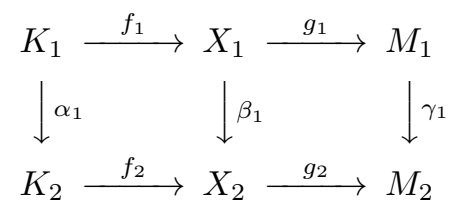

is commutative.

3. Composition of morphisms:

Let $\bar{K}_{1}=\left(K_{1}, L, M_{1}\right), \bar{K}_{2}=\left(K_{2}, L, M_{2}\right), \bar{K}_{3}=$ $\left(K_{3}, L, M_{3}\right) \in \operatorname{Obj}\left(\mathcal{C}_{L}\right)$,

$\theta_{1}=\left(\alpha_{1}, \beta_{1}, \gamma_{1}\right) \in \operatorname{Mor}_{\mathcal{C}_{L}}\left(\bar{K}_{1}, \bar{K}_{2}\right)$, and

$\theta_{2}=\left(\alpha_{2}, \beta_{2}, \gamma_{2}\right) \in \operatorname{Mor}_{\mathcal{C}_{L}}\left(\bar{K}_{2}, \bar{K}_{3}\right)$.

Hence, we have the following commutative diagrams:

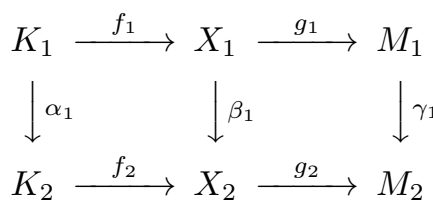

and

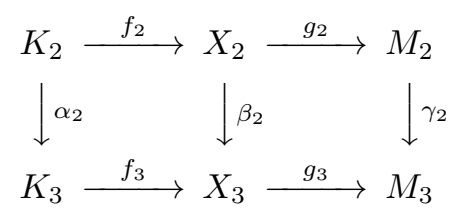

Then $\theta_{3}=\left(\alpha_{2} \circ \alpha_{1}, \beta_{2} \circ \beta_{1}, \gamma_{2} \circ \gamma_{1}\right)$ is a morphism from $\bar{K}_{2}$ to $\bar{K}_{3}$. We can see this in the following commutative 
diagram with exact rows:

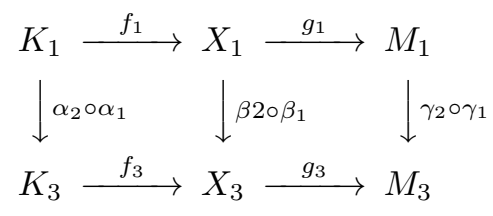

Then, we will check whether the morphisms hold associative law.

Let $\bar{K}_{1}=\left(K_{1}, L, M_{1}\right), \bar{K}_{2}=\left(K_{2}, L, M_{2}\right), \bar{K}_{3}=$ $\left(K_{3}, L, M_{3}\right)$ and $\bar{K}_{4}=\left(K_{4}, L, M_{4}\right)$ are objects in $\mathcal{C}_{L}$, $\theta_{1} \in \operatorname{Mor}_{\mathcal{C}_{L}}\left(\bar{K}_{1}, \bar{K}_{2}\right), \theta_{2} \in \operatorname{Mor}_{\mathcal{C}_{L}}\left(\bar{K}_{2}, \bar{K}_{3}\right), \theta_{3} \in$ $\operatorname{Mor}_{\mathcal{C}_{L}}\left(\bar{K}_{3}, \bar{K}_{4}\right)$. Then,

$$
\begin{aligned}
\theta_{3} \circ_{c}\left(\theta_{2} \circ_{c} \theta_{1}\right) & =\left(\alpha_{3}, \beta_{3}, \gamma_{3}\right) \circ_{c}\left(\left(\alpha_{2}, \beta_{2}, \gamma_{2}\right) \circ_{c}\left(\alpha_{1}, \beta_{1}, \gamma_{1}\right)\right. \\
& =\left(\alpha_{3}, \beta_{3}, \gamma_{3}\right) \circ_{c}\left(\alpha_{2} \circ \alpha_{1}, \beta_{2} \circ \beta_{1}, \gamma_{2} \circ \gamma_{1}\right) \\
& =\left(\alpha_{3} \circ \alpha_{2} \circ \alpha_{1}, \beta_{3} \circ \beta_{2} \circ \beta_{1}, \gamma_{3} \circ \gamma_{2} \circ \gamma_{1}\right) \\
& =\left(\alpha_{3} \circ \alpha_{2}, \beta_{3} \circ \beta_{2}, \gamma_{3} \circ \gamma_{2}\right) \circ_{c}\left(\alpha_{1}, \beta_{1}, \gamma_{1}\right) \\
& =\left(\left(\alpha_{3}, \beta_{3}, \gamma_{3}\right) \circ_{c}\left(\alpha_{2}, \beta_{2}, \gamma_{2}\right)\right) \circ_{c}\left(\alpha_{1}, \beta_{1}, \gamma_{1}\right) \\
& =\left(\theta_{3} \circ{ }_{c} \theta_{2}\right) \circ_{c} \theta_{1} .
\end{aligned}
$$

Hence, morphisms of category of $\mathcal{C}_{L}$ hold associative law, i.e

$$
\theta_{3} \circ_{c}\left(\theta_{2} \circ_{c} \theta_{1}\right)=\left(\theta_{3} \circ_{c} \theta_{2}\right) \circ_{c} \theta_{1},
$$

for every $\theta_{1} \in \operatorname{Mor}_{\mathcal{C}_{X}}\left(\bar{K}_{1}, \bar{K}_{2}\right), \theta_{2} \in \operatorname{Mor}_{\mathcal{C}_{X}}\left(\bar{K}_{2}, \bar{K}_{3}\right)$, $\theta_{3} \in \operatorname{Mor}_{\mathcal{C}_{X}}\left(\bar{K}_{3}, \bar{K}_{4}\right)$.

For every $\bar{K}=(K, L, M) \in \operatorname{Obj}\left(\mathcal{C}_{L}\right)$, there is a morphism $i d_{\bar{K}}=\left(i d_{K}, i d_{X}, i d_{M}\right)$ in $\operatorname{Mor}_{C_{L}}(\bar{K}, \bar{K})$, the identity of $\bar{K}$, with

$$
\theta \circ_{c} i d_{\bar{K}}=i d_{\bar{K}_{1}} \circ_{c} \theta=\theta,
$$

for every $\theta \in \operatorname{Mor}_{C_{X}}\left(\bar{K}, \bar{K}_{1}\right), \bar{K}_{1}=\left(K_{1}, L, M_{1}\right) \in$ $\operatorname{Obj}\left(\mathcal{C}_{L}\right)$.

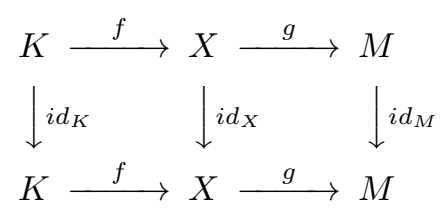

So, we can conclude that $\mathcal{C}_{L}$ is a category.

In the following proposition, we will show that if $\mathrm{L}$ is a uniserial $R$-module, then a category $\mathcal{C}_{L}$ is pre-additive.

Proposition 1 Let $L$ be a uniserial module. The category $\mathcal{C}_{L}$ is a pre-additive category.

\section{Proof.}

1. Let the triples $\bar{K}_{1}=\left(K_{1}, L, M_{1}\right)$ and $\bar{K}_{2}=\left(K_{2}, L, M_{2}\right)$ are objects in $\mathcal{C}_{L}$.

Then, there are submodules $X_{1}$ and $X_{2}$ of $L$, where $X_{1}$ and $X_{2}$ are maximal element of $\sigma\left(K_{1}, L, M_{1}\right)$ and $\sigma\left(K_{2}, L, M_{2}\right)$, respectively, such that the sequences

$$
K_{1} \stackrel{f_{1}}{\longrightarrow} X_{1} \stackrel{g_{1}}{\longrightarrow} M_{1}
$$

and

$$
K_{2} \stackrel{f_{2}}{\longrightarrow} X_{2} \stackrel{g_{2}}{\longrightarrow} M_{2}
$$

are exact.

We define:

$\left(\alpha_{1}, \beta_{1}, \gamma_{1}\right)+{ }_{c}\left(\alpha_{2}, \beta_{2}, \gamma_{2}\right)=\left(\alpha_{1}+\alpha_{2}, \beta_{1}+\beta_{2}, \gamma_{1}+\gamma_{2}\right)$,

for all $\left(\alpha_{1}, \beta_{1}, \gamma_{1}\right),\left(\alpha_{2}, \beta_{2}, \gamma_{2}\right) \in \operatorname{Hom}_{\mathcal{C}_{X}}\left(\bar{K}_{1}, \bar{K}_{2}\right)$.

It is easy to see that $\left(\operatorname{Hom}_{\mathcal{C}_{X}}\left(\bar{K}_{1}, \bar{K}_{2}\right),+_{c}\right)$ is an Abelian group and the composition of morphisms

$$
\operatorname{Hom}_{\mathcal{C}_{L}}\left(\bar{K}_{2}, \bar{K}_{3}\right) \times \operatorname{Hom}_{\mathcal{C}_{L}}\left(\bar{K}_{1}, \bar{K}_{2}\right) \rightarrow \operatorname{Hom}_{\mathcal{C}_{L}}\left(\bar{K}_{1}, \bar{K}_{3}\right)
$$

is bilinear, i.e.

$$
\begin{array}{r}
\left(\left(\alpha_{1}, \beta_{1}, \gamma_{1}\right)+_{c}\left(\alpha_{2}, \beta_{2}, \gamma_{2}\right)\right) \circ_{c}(f, g, h) \\
=\left(\left(\alpha_{1}, \beta_{1}, \gamma_{1}\right) \circ_{c}(f, g, h)\right)+_{c} \\
\left(\left(\alpha_{2}, \beta_{2}, \gamma_{2}\right) \circ_{c}(f, g, h)\right)
\end{array}
$$

and

$$
\begin{array}{r}
\left(f^{\prime}, g^{\prime}, h^{\prime}\right) \circ_{c}\left(\left(\alpha_{1}, \beta_{1}, \gamma_{1}\right)+_{c}\left(\alpha_{2}, \beta_{2}, \gamma_{2}\right)\right) \\
=\left(\left(f^{\prime}, g^{\prime}, h^{\prime}\right) \circ_{c}\left(\alpha_{1}, \beta_{1}, \gamma_{1}\right)\right)+_{c} \\
\left(\left(f^{\prime}, g^{\prime}, h^{\prime}\right) \circ_{c}\left(\alpha_{2}, \beta_{2}, \gamma_{2}\right)\right)
\end{array}
$$

2. The zero object in $\mathcal{C}_{L}$ is triple $(0,0,0)$.

Hence, the category $\mathcal{C}_{L}$ is a pre-additive category.

Let $L$ be a uniserial module, and $Y$ be a submodule of $L$. Then we can construct the category $\mathcal{C}_{L}$ and $\mathcal{C}_{Y}$. Since every object in $\mathcal{C}_{Y}$ is an object in $\mathcal{C}_{L}$, we have the following proposition.

Proposition 2 Let $L$ be a uniserial module, and $Y$ be a submodule of $L$. Then $\mathcal{C}_{Y}$ is a full subcategory of $\mathcal{C}_{L}$.

We recall that the sequence $0 \rightarrow M_{1} \stackrel{\phi}{\rightarrow} M \stackrel{\psi}{\rightarrow} M_{2}$ is exact if and only if the sequence: $0 \rightarrow \operatorname{Hom}_{R}\left(N, M_{1}\right) \stackrel{\phi_{\star}}{\longrightarrow}$ $\operatorname{Hom}_{R}(N, M) \stackrel{\psi_{\star}}{\longrightarrow} \operatorname{Hom}_{R}\left(N, M_{2}\right)$ is an exact sequence of $\mathbb{Z}$-modules for all $R$-modules $N$. The sequence $M_{1} \stackrel{\phi}{\rightarrow} M \stackrel{\psi}{\rightarrow}$ $M_{2} \rightarrow 0$ is exact if and only if the sequence:

$0 \rightarrow \operatorname{Hom}_{R}\left(M_{2}, N\right) \stackrel{\psi^{\star}}{\longrightarrow} \operatorname{Hom}_{R}(M, N) \stackrel{\phi^{\star}}{\longrightarrow} \operatorname{Hom}_{R}\left(M_{1}, N\right)$

is an exact sequence of $\mathbb{Z}$-modules for all $R$-modules $N$ [1]. Next, we will investigate whether the Hom-functor preserves the sub-exactness. Now, we define a monic $X$-sub-exact and epic $X$-sub-exact as follow:

Definition 1 Let $K, L, M$ be R-modules and $X$ be a submodule of $L$. Then the triple $(K, L, M)$ is said to be a monic $X$ sub-exact at $L$ if there exist $R$-homomorphisms $f$ and $g$ such that the sequence:

$$
K \stackrel{f}{\rightarrow} X \stackrel{g}{\rightarrow} M
$$

is an exact sequence and $f$ is a monomorphism.

The triple $(K, L, M)$ is said to be an epic $X$-sub-exact at $L$ if there exist $R$-homomorphisms $f$ and $g$ such that the sequence of R-modules and R-homomorphisms:

$$
K \stackrel{f}{\rightarrow} X \stackrel{g}{\rightarrow} M
$$

is an exact sequence and $g$ is an epimorphism. 
Next, we will prove that a monic $X$-sub-exactness of $(K, L, M)$ implies a monic $H_{R}(N, X)$-sub-exactness of $\left(H_{R}(N, K), H_{R}(N, L), H_{R}(N, M)\right)$, for any $R$ module $N$.

Proposition 3 Let $K, L, M$ be R-modules and $X$ be a submodule of $L$. The triple $(K, L, M)$ is a monic $X$-sub-exact, $i$. e. the sequence

$$
K \stackrel{f}{\rightarrow} X \stackrel{g}{\rightarrow} M
$$

is exact at $X$ and $f$ is a monomorphism, if and only if the triple $\mathbb{Z}$-modules :

$$
\left(\operatorname{Hom}_{R}(N, K), \operatorname{Hom}_{R}(N, L), \operatorname{Hom}_{R}(N, M)\right)
$$

is a monic $\operatorname{Hom}_{R}(N, X)$-sub-exact, for all R-modules $N$.

Proof. The triple $(K, L, M)$ is a monic $X$-sub-exact, i.e the sequence $K \stackrel{f}{\rightarrow} X \stackrel{g}{\rightarrow} M$ is exact at $X$ and $f$ is a monomorphism, for any $R$-module $N$, if and only if the sequence of $\mathbb{Z}$-modules:

$$
\operatorname{Hom}_{R}(N, K) \stackrel{f_{\star}}{\longrightarrow} \operatorname{Hom}_{R}(N, X) \stackrel{g_{\star}}{\longrightarrow} \operatorname{Hom}_{R}(N, M)
$$

is exact at $\operatorname{Hom}_{R}(N, X)$ and $f_{\star}$ is a monomorphism.

Furthermore, for any $h \in \operatorname{Hom}_{R}(N, X), h \in$ $H_{\text {om }}(N, L)$. Hence,

$\operatorname{Hom}_{R}(N, X) \subseteq \operatorname{Hom}_{R}(N, L)$. So, we can conclude that the triple $(K, L, M)$ is a monic $X$-sub-exact, i. e. the sequence $K \stackrel{f}{\rightarrow} X \stackrel{g}{\rightarrow} M$ is exact at $X$ and $f$ is a monomorphism, if and only if the triple $\mathbb{Z}$-modules :

$$
\left(\operatorname{Hom}_{R}(N, K), \operatorname{Hom}_{R}(N, L), \operatorname{Hom}_{R}(N, M)\right)
$$

is a monic $H_{R}(N, X)$-sub-exact, for all $R$-modules $N$.

On the other hand, we will investigate whether the triple:

$$
\left(\operatorname{Hom}_{R}(M, N),\left(\operatorname{Hom}_{R}(L, N),\left(\operatorname{Hom}_{R}(K, N)\right)\right.\right.
$$

is also a $\left(\operatorname{Hom}_{R}(X, N)\right.$-sub-exact, for all $R$-modules $N$. If $h \in \operatorname{Hom}_{R}(X, N)$, then $h$ is not necessary an element of $\operatorname{Hom}_{R}(L, N)$. For example, the inclusion $i \in \operatorname{Hom}_{\mathbb{Z}}(\mathbb{Z}, \mathbb{Z})$, but $i \notin H o m_{\mathbb{Z}}(\mathbb{Q}, \mathbb{Z})$.

In the following proposition, we provide a necessary condition to a submodule $X$ of $L$ so that the triple $\left(H_{R}(M, N),\left(H_{R} m_{R}(L, N),\left(H_{R}(K, N)\right)\right.\right.$ is a $\left(H_{R} m_{R}(X, N)\right.$-sub-exact, for all $R$-module $N$.

Proposition 4 Let $K, L, M$ be R-modules and $X$ be a direct summand of $L$. The triple $(K, L, M)$ is an epic $X$-sub-exact, i. e. the sequence

$$
K \stackrel{f}{\rightarrow} X \stackrel{g}{\rightarrow} M
$$

is exact at $X$ and $g$ is an epimorphism, if and only if the triple $\mathbb{Z}$-modules:

$$
\left(\operatorname{Hom}_{R}(M, N), \operatorname{Hom}_{R}(L, N), \operatorname{Hom}_{R}(K, N)\right)
$$

is a monic $\operatorname{Hom}_{R}(X, N)$-sub-exact, for all R-module $N$.
Proof. The triple $(K, L, M)$ is an epic $X$-sub-exact, i.e the sequence

$$
K \stackrel{f}{\rightarrow} X \stackrel{g}{\rightarrow} M
$$

is exact at $X$ and $g$ is an epimorphism, if and only if the sequence of $\mathbb{Z}$-modules:

$$
\operatorname{Hom}_{R}(M, N) \stackrel{g^{\star}}{\longrightarrow} \operatorname{Hom}_{R}(X, N) \stackrel{f_{\star}}{\longrightarrow} \operatorname{Hom}_{R}(M, N)
$$

is a monic $\operatorname{Hom}_{R}(X, N)$-sub-exact.

Since $X$ is a direct summand of $L$, there is a submodule $Y$ of $L$ such that $L \simeq X \oplus Y$. Let $h \in \operatorname{Hom}_{R}(X, N)$. We can define a homomorphism

$$
h^{\prime}: L \rightarrow N,
$$

where:

$$
h^{\prime}(a)= \begin{cases}h(a) & ; \text { if } a \in X, \\ 0 & ; \text { otherwise. }\end{cases}
$$

We will show that $h^{\prime}$ is an $R$-homomorphism from $L$ to $N$. Let $a, b \in L$ and $r \in R$. We have $a=x_{1}+y_{1}$ and $b=x_{2}+y_{2}$, where $x_{1}, x_{2} \in X$ and $y_{1}, y_{2} \in Y$. Therefore, we get:

$$
\begin{aligned}
f^{\prime}(a+b) & =f^{\prime}\left(\left(x_{1}+y_{1}\right)+\left(x_{2}+y_{2}\right)\right) \\
& =f^{\prime}\left(\left(x_{1}+x_{2}\right)+\left(y_{1}+y_{2}\right)\right) \\
& =f\left(x_{1}+x_{2}\right) \\
& =f\left(x_{1}\right)+f\left(x_{2}\right) \\
& =f^{\prime}\left(x_{1}+y_{1}\right)+f^{\prime}\left(x_{2}+y_{2}\right) \\
& =f^{\prime}(a)+f^{\prime}(b) .
\end{aligned}
$$

and

$$
\begin{aligned}
f^{\prime}(r a) & =f^{\prime}(r(x+y)) \\
& =f^{\prime}(r x+r y) \\
& =f(r x)=r f(x) \\
& =r f^{\prime}(a) .
\end{aligned}
$$

We can conclude that $h^{\prime}$ is an $R$-homomorphism from $L$ to $N$.

So, for every $h \in \operatorname{Hom}_{R}(X, N)$, we can define an $R$ homomorphism $h^{\prime} \in \operatorname{Hom}_{R}(L, N)$. Therefore, there exists a monomorphism

$$
\theta: \operatorname{Hom}_{R}(X, N) \rightarrow \operatorname{Hom}_{R}(L, N),
$$

where $\theta(h)=h^{\prime}$. We have $\operatorname{Hom}_{R}(X, N)$ is isomorphic to a submodule of $\operatorname{Hom}_{R}(L, N)$. Consequently, the triple $\mathbb{Z}$ modules:

$$
\left(\operatorname{Hom}_{R}(M, N), \operatorname{Hom}_{R}(L, N), \operatorname{Hom}_{R}(K, N)\right)
$$

is a monic $\operatorname{Hom}_{R}(X, N)$-sub-exact, for all $R$-modules $N$. 
Consider now the family of monic $X$-sub-exact sequences, where $X$ is a submodule of a uniserial module $L$, as follow:

$\operatorname{Obj}\left(\mathcal{C}_{L}^{\star}\right)=\{(K, L, M) \mid(K, L, M)$ is a monic $X$-sub-exact $\}$

It is clear that $\operatorname{Obj}\left(\mathcal{C}_{L}^{\star}\right) \subseteq \operatorname{Obj}\left(\mathcal{C}_{L}\right)$.

In Proposition 1, we proved that $C_{L}$ is a pre-additive category. According to [11], an Abelian category is an additive category in which every morphism has kernel and cokernel, and for every morphism $f: X \rightarrow Y$, the natural morphism coim $f \rightarrow i m f$ is an isomorphism. We will show that every morphism in $\mathcal{C}_{L}^{\star}$ has a kernel.

Proposition 5 Let $L$ be a uniserial module. Then every morphism in $\mathcal{C}_{L}^{\star}$ has a kernel.

Proof. Let $(\alpha, \beta, \gamma) \in \operatorname{Hom}\left(\left(K_{1}, L, M_{1}\right),\left(K_{2}, L, M_{2}\right)\right)$. Then, there are submodules $X_{1}, X_{2}$ of $L$, where $X_{1}$ and $X_{2}$ are maximal element of $\sigma\left(K_{1}, L, M_{1}\right)$ and $\sigma\left(K_{2}, L, M_{2}\right)$, respectively, such that the following sequences:

$$
K_{1} \stackrel{f_{1}}{\longrightarrow} X_{1} \stackrel{g_{1}}{\longrightarrow} M_{1}
$$

and

$$
K_{2} \stackrel{f_{2}}{\longrightarrow} X_{2} \stackrel{g_{2}}{\longrightarrow} M_{2}
$$

are exact, where $f_{1}, f_{2}$ are monomorphisms. We have the following diagram:

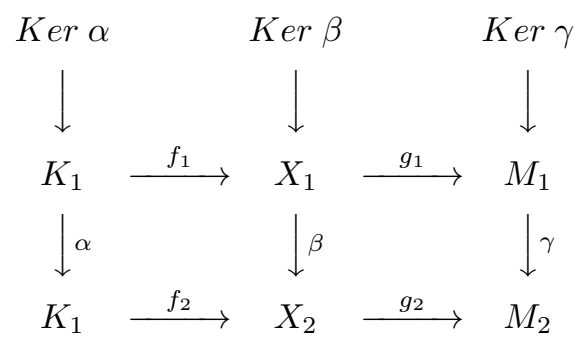

Since $f_{1}, f_{2}$ are monomorphisms, then by Snake Lemma, the first row, i.e. $\operatorname{Ker} \alpha \rightarrow \operatorname{Ker} \beta \rightarrow \operatorname{Ker} \gamma$ is exact. So, (Ker $\alpha, L, \operatorname{Ker} \gamma)$ is in $\mathcal{C}_{L}^{\star}$ and it is kernel of $(\alpha, \beta, \gamma)$.

Since a module factor of $L$ is not a submodule of $L$, every morphism in a category $\mathcal{C}_{L}$ does not have a cokernel. So, $\mathcal{C}_{L}$ is not an abelian category.

\section{Conclusions}

For any uniserial $R$-module $L$, we can construct a category $\mathcal{C}_{L}$. The object of a category $\mathcal{C}_{L}$ is triple $(K, L, M)$ such that $(K, L, M)$ is an $X$-sub-exact sequence, where $X$ is the maximal element of the set of all submodules $Y$ of $L$ such that $(K, L, M)$ is a $Y$-sub-exact. We proved that $\mathcal{C}_{L}$ is a preadditive category, a category $\mathcal{C}_{Y}$ is a full subcategory of $\mathcal{C}_{L}$, for any submodule $Y$ of $L$. Every morphism in $\mathcal{C}_{L}^{\star}$ has a kernel.

Furthermore, we proved that a monic $X$-subexactness of $(K, L, M)$ implies a monic sub-exactness of $\quad\left(\operatorname{Hom}_{R}(N, K), \operatorname{Hom}_{R}(N, L), \operatorname{Hom}_{R}(N, M)\right)$ If $X$ is a direct summand of $L$, then an epic $X$-subexactness of $(K, L, M)$ implies a monic sub-exactness of $\left(\operatorname{Hom}_{R}(M, N), \operatorname{Hom}_{R}(L, N), \operatorname{Hom}_{R}(K, N)\right)$, for any $R$-module $N$.

\section{Acknowledgements}

We are very grateful to experts for their appropriate and constructive suggestions to improve this manuscript. More-

over, we wish to thank the Research Institutions and Community Service of Universitas Lampung for this research support and funding under the Research Contract No: 1667/UN 26.21/PN/2021.

\section{REFERENCES}

[1] Adkins, W. A., Weintraub, S. H., "Algebra: An Approach via Module Theory," Springer-Verlag New York, Inc., USA., 1992.

[2] Anvariyeh, S. M., Davvaz, B., "On Quasi-Exact Sequences," Bull. Korean Math. Soc., vol. 42, no. 1, 149-155, 2005. https://doi.org/10.4134/BKMS.2005.42.1.149

[3] Anvariyeh, S. M., Davvaz, B., "U-Split-Exact Sequences," Far East J. Math. Sci. (FJMS), vol. 4, no.2, 209-219, 2002.

[4] Davvaz, B., Shabani-Solt, H., "A Generalization of Homological Algebra," J. Korean Math. Soc., vol. 39, no.6, 881-898, 2002.

https://doi.org/10.4134/JKMS.2002.39.6.881

[5] Davvaz, B., Parnian-Garamaleky, Y. A., "A Note on Exact Sequences," Bull.Malays. Math. Sci. Soc., vol. 22, no. 1, 53-56, 1999.

[6] Faisol, A., Fitriani, Sifriyani, "Determining the Noetherian Property of Generalized Power Series Modules by Using XSub-Exact Sequence," J. Phys. Conf. Series, 1751, 012028, 2021. doi:10.1088/1742-6596/1751/1/012028

[7] Fitriani, Surodjo, B., Wijayanti, I. E., On X-sub-exact Sequences, Far East J. Math. Sci. (FJMS), vol. 100, no. 7, 10551065, 2016.

http://dx.doi.org/10.17654/MS100071055

[8] Fitriani, Wijayanti, I. E., Surodjo, B., "On X-sub-linearly Independent Modules," J. Phys. Conf. Series, 893, 2017. doi: 10.1088/1742-6596/893/1/012008

[9] Fitriani, Wijayanti, I. E., Surodjo,B., "A Generalization of Basis and Free Modules Relatives to a Family $\mathcal{U}$ of $R$-Modules," J. Phys. Conf. Series, 1097, 012087, 2018. doi: 10.1088/1742$6596 / 1097 / 1 / 012087$

[10] Fitriani, Wijayanti, I. E., Surodjo, B., "Generalization of $U$-Generator and $M$-Subgenerator Related to Category $\sigma[M]$," J. Math. Res., vol. 10, no. 4, 101-106, 2018. https://doi.org/10.5539/jmr.v10n4p101

[11] Holm, T., Jorgensen, P., Rouquier, R., "Triangulated Categories," Cambridge University Press, New York, USA, 2010.

[12] Lam, T. Y., "A First Course in Noncommutative Rings," $2^{\text {nd }}$ ed., Springer-Verlag, New York, 2001.

[13] Wisbauer, R., "Foundation of Module and Ring Theory," Gordon and Breach, Philadelphia, USA, 1991. 